\title{
Uso do Verapamil em Gestantes Hipertensas Crônicas. Análise do Fluxo das Artérias Uterinas e Umbilical
}

\author{
Use of Verapamil in Chronic Hypertensive Pregnant Women. Flow \\ Analysis of Uterine Arteries and Umbilical Artery
}

\begin{abstract}
Marcus Jose do Amaral Vasconcellos ${ }^{1}$, Hermógenes Chaves Netto ${ }^{2}$ Soubhi Kahhale ${ }^{3}$, Paulo Jorge Almeida ${ }^{2}$
\end{abstract}

\begin{abstract}
RESUMO
Objetivo: este trabalho, utilizando verapamil, um bloqueador dos canais lentos de cálcio, constituiu ensaio clínico randomizado, duplo-cego e placebo controlado, e objetivou procurar variação do fluxo uteroplacentário e fetoplacentário durante uso oral crônico do fármaco em gestantes com hipertensão crônica leve para moderada.

Métodos: 123 pacientes divididas em dois grupos: grupo estudo $(n=61)$, submetidas a 240 $\mathrm{mg} /$ dia de verapamil, e grupo controle $(n=62)$, submetidas ao placebo. As pacientes randomizadas em grupos de quatro utilizaram a medicação ou placebo durante trinta dias. Um exame do fluxo das artérias uterinas e da artéria umbilical pela dopplervelocimetria foi registrado. Pelo cálculo da média e desvio padrão, foram comparados os valores dos indices de resistência (IR) e pulsatilidade (IP) e da relação sístole/diástole $(A / B)$ das artérias em estudo após administração dos comprimidos.

Resultados: o grupo verapamil apresentou os seguintes valores médios para as artérias uterinas: $I R=0,82(0,28)$, IP de 1,06 $(0,12)$ e $A / B$ de 2,42 (0,51). O grupo placebo mostrou: IR de 0,75 (0,35), IP de 1,00 (0,18) e A/B de 2,30 (0,38). Quando analisada a artéria umbilical, os valores foram para o grupo verapamil: $I R=0,73(0,12), I P=1,04(0,13)$ e $A / B=2,94$ (0,32). No grupo placebo, $I R=0,70(0,14), I P=1,03(0,07)$ e $A / B=3,02(0,78)$. A análise estatística das diferenças das médias por meio da razão $F$ mostrou não haver diferença entre os dois grupos avaliados.

Conclusão: este trabalho referenda o uso do verapamil entre gestantes com hipertensão crônica (leve para moderada), pois não oferece prejuizos no fluxo uteroplacentário $e$ fetoplacentário.
\end{abstract}

PALAVRAS-CHAVE: Hipertensão na Gravidez. Verapamil. Fluxo Placentário. Dopplervelocimetria.

\section{Introdução}

As ações de saúde inerentes às sindromes hipertensivas devem levar em conta as elevadas taxas de mortalidade materna, que hoje já atingem mais de 140/100.000 nascidos vivos em nos-

${ }^{1}$ Disciplina de Obstetrícia - Universidade do Rio de Janeiro

${ }^{2}$ Clínica Obstétrica - Universidade Federal do Rio de Janeiro

${ }^{3}$ Clínica Obstétrica - Faculdade de Medicina da Universidade de São Paulo

Correspondência

Marcus Jose do Amaral Vasconcellos

Rua Esteves Júnior, 64/501 - Laranjeiras

22231-160 - Rio de Janeiro - RJ so país. Quando está em tela a mortalidade fetal e neonatal precoce, cifras de 150/1.000 assustam obstetras e neonatologistas. Estas afirmativas fazem parte de documentos oficiais do Ministério da Saúde, disponiveis no endereço eletrônico do órgão (http: / / www.saude.gov.br/programas/mulher/dados.htm).

Além disto, quando abordamos a necessidade de tratamento de uma síndrome hipertensiva, concordamos com alguns paradigmas ressaltados por Henriksen ${ }^{1}$, principalmente com o último: evitar complicações maternas graves como a eclampsia, a sindrome HELLP, a hemorragia cerebral, os acidentes anestésicos e a falência de 
múltiplos órgãos; evitar a progressão de quadros de pré-eclampsia; evitar a sobreposição de préeclampsia em paciente portadora de doença hipertensiva vascular crônica prévia à gestação; procurar um melhor resultado perinatal afastando o sofrimento fetal e a mortalidade neonatal; atingir a viabilidade e o peso adequado do recémnato; atingir um bom prognóstico no desenvolvimento dos filhos de mãe hipertensa; proporcionar, a longo prazo, um baixo risco cardiovascular e renal materno.

Kaplan ${ }^{2}$ cita duas metanálises, que com clareza mostram que o risco relativo de acidente vascular cerebral e doença coronariana, na população em geral, era significativamente crescente na medida que também cresciam os valores da pressão arterial diastólica. Quando utilizaram o nivel discriminatório de $91 \mathrm{mmHg}$, os autores encontraram um risco relativo duas vezes maior para valores de $98 \mathrm{mmHg}$ e quatro vezes quando a pressão diastólica está acima de $105 \mathrm{mmHg}$.

As evidências referidas nestas duas metanálises podem ser transportadas para nossa gestante hipertensa crônica. A sobrecarga cardíaca, o aumento da resistência vascular periférica, o aumento do volume circulante, eventos marcantes no ciclo gravídico-puerperal, certamente nos fazem pensar: adotar conduta ativa trará uma proteção para o futuro desta mulher?

A conduta ativa, sob a ótica deste raciocínio de custo e beneficio, nos parece mais adequada para a nossa realidade mesmo quando particularizamos as mulheres grávidas. A visão emocional que é sugerida, com a estigmatização da paciente como "hipertensa", ao nosso ver, tem muitos aspectos benéficos. Certamente a conscientização da gestante brasileira quanto à suas limitações, além de incentivo a mudanças no seu estilo de vida, será vantajosa para o seu prognóstico.

O enfoque financeiro compara o custo das drogas anti-hipertensivas com gastos com complicações. Também sob este ponto de vista a realidade nacional parece nos conduzir a uma constatação, que mesmo não sendo a mais correta, é a que vivenciamos: é muito mais caro uma internação com características de emergência do que a utilização de drogas de rotina.

Contribuição para esta polêmica foi dada pela Canadian Hypertension Society, relatada na publicação de Moutquin et al. ${ }^{3}$, ao admitir que $90 \%$ das gestantes canadenses hipertensas crônicas com caracteristicas leve e moderada evoluem com bom prognóstico materno e perinatal. As que compõem os $10 \%$ restantes são consideradas de alto risco, merecendo abordagem anti-hipertensiva. Estas pacientes, mesmo com niveis de pressão arterial pouco elevados, podem ter órgãos nobres atingidos, e junto com outros fatores de risco como idade avançada, história de hipertensão arterial com mais de 15 anos de duração, história prévia de crescimento retardado ou morte perinatal, fazem parte do grupo que merece atendimento individualizado.

O que nos parece mais importante neste consenso canadense está firmado em curto parágrafo, que passamos a reproduzir : “. . . algumas mulheres não permitem uma conduta não-medicamentosa por razões socioeconômicas, prole extensa, necessidade de trabalho extradomiciliar fatigante $e$ estresse constante".

Esta visão está perfeitamente inserida na nossa realidade, pois o perfil social de nossa gestante a transforma em paciente de risco. As dificuldades enfrentadas, às vezes com tripla jornada de trabalho, colaboram para agravamento da hipertensão arterial. As recomendações finais do documento preconizam conduta não-farmacológica (repouso, visita domiciliar, internação liberal) nas pacientes consideradas portadoras de hipertensão arterial crônica leve e moderada, mas abre uma perspectiva: em vigência de fatores de risco maternos, a utilização dos anti-hipertensivos deve ser pensada.

As considerações feitas pelos canadenses são muito parecidas para norte-americanos e australianos. Em normas do National High Blood Pressure Education Program Working Group Report (Estados Unidos) ${ }^{4}$ e da Australian Society for the Study of Hypertension in Pregnancy ${ }^{5}$, a internação para pacientes hipertensas é recomendada apesar de não serem conclusivos os beneficios desta conduta. Ambos os documentos enfatizam que o manuseio não-farmacológico domiciliar está admitido desde que a gestação da paciente possa transcorrer em condições de estabilidade emocional, fisica, dietética e com acesso facilitado ao prénatal, inclusive com atendimento domiciliar. $\mathrm{Na}$ ausência destas premissas a internação hospitalar será quase que obrigatória.

A reflexão que imediatamente fazemos, de posse destes três documentos da literatura internacional, é que nossa estrutura de saúde pública, além de nossas condições sociais, em nada permitem que repouso compulsório, visita domiciliar e internação alargada possam ser adotados como medida protocolar na condução destas gestantes.

Com finalidade de justificar nossa proposta de trabalho, citamos contribuição significativa sobre o impacto da hipertensão crônica sobre o concepto dada por Jain ${ }^{6}$. Por meio de estudo retrospectivo no Departamento de Pediatria da Universidade de Chicago (1982-1987), catalogaram 110.000 partos consecutivos. Deste montante, 8.019 pacientes cursaram com uma sindrome 
hipertensiva. Entre estas, 5.971 apresentaram hipertensão induzida pela gestação e 2.048 foram diagnosticadas como hipertensas crônicas. Os dois grupos foram comparados e os resultados mostraram maior incidência de morte fetal, prematuridade, baixo peso do recém-nato e crescimento retardado no grupo de hipertensas crônicas.

Kahhale e Zugaib ${ }^{7}$, em seu capítulo sobre a gestante hipertensa crônica, são favoráveis ao tratamento ativo com drogas nestas pacientes, recomendando os $\beta$-bloqueadores como fármaco de escolha. Justificam sua posição pelos altos indices de morte e acidentes coronarianos no futuro destas mulheres, além de acreditarem que a diminuição dos níveis tensionais será benéfica para a gestação em curso.

Lindheimer et al. $^{8}$, na nova edição do livro texto clássico de Chesley, são categóricos em afirmar que as gestantes hipertensas crônicas experimentam uma aceleração no quadro vascular, podendo prejudicar órgãos nobres como o cérebro, coração e rins.

Com estas ponderações, acrescidas de outras opiniões, metanálises e consensos internacionais, justificamos a conduta ativa medicamentosa na gestante hipertensa crônica, mesmo em suas formas leve e moderada. O que discutimos em seguida é como viabilizar esta opção: qual droga utilizar? qual a repercussão deste fármaco sobre a circulação uteroplacentária e fetoplacentária, e conseqüentemente sobre o crescimento e a vitalidade do concepto? estaremos tratando a mãe com prejuizo fetal e neonatal?

Os bloqueadores dos canais de cálcio vêm ganhando espaço crescente no arsenal terapêutico envolvido no controle da gestante hipertensa. Para o entendimento do mecanismo de ação dos bloqueadores dos canais de cálcio devemos ter em mente que existem três tipos de canais de cálcio na membrana celular: canais voltagem-dependentes ("long-acting" - ação lenta), canais transitórios e canais neuronais. Os bloqueadores de canais de cálcio atuam nos canais de ação lenta predominantes no miocárdio e vasculatura, permitindo impacto na contratilidade cardíaca e dos vasos ${ }^{9}$.

$\mathrm{Na}$ fibra miocárdica pode-se controlar farmacologicamente a condutividade do cálcio através da membrana. As substâncias estimulantes dos receptores $\beta$-adrenérgicos como a epinefrina, norepinefrina e isoproterenol aumentam seletivamente o aporte de cálcio pela membrana celular miocárdica excitada, aumentando a força contrátil.

Os antagonistas do cálcio exercem efeito exatamente oposto. Estas drogas restringem a contração miocárdica levando a uma redução dosedependente da entrada de cálcio na célula. Com este mecanismo básico estes fármacos atuam na hipercinesia cardíaca e apresentam marcante efeito relaxante da musculatura lisa, particularmente nos vasos, exercendo efeitos vasodilatador e espasmolítico na circulação sistêmica e coronariana. Um efeito secundário tocolítico e broncodilatador deve ser ressaltado na vigência do uso dos bloqueadores da entrada de cálcio na célula muscular.

Uma outra ação deste grupo de fármacos é sua interferência na função renal, promovendo uma melhor perfusão no órgão ${ }^{9}$. Este estudo mostra que os bloqueadores dos canais de cálcio controlam a hipertrofia renal, modulam o trânsito das macromoléculas através do mesângio, melhoram a nefrocalcinose urêmica, atenuam os efeitos mitogênicos dos fatores de crescimento, como o fator ativador plaquetário e o fator derivado do crescimento plaquetário, e exercem inibição da entrada do cálcio na célula. Com certeza este efeito será benéfico para a gestante.

Este tipo de droga mostra-se útil no tratamento da hipertensão porque, em contraste com outros hipotensores, apresenta efeito vasodilatador coronariano, melhorando a perfusão subendocárdica, e causa relaxamento ventricular. Em estudos mais recentes, Frishman e Michaelson ${ }^{10}$ concordaram com todos estes efeitos, mas chamaram a atenção para um possivel estimulo de reflexo simpático levando a acidentes cardiacos. Estes autores ainda não aceitam o bloqueador de canais de cálcio como droga de primeira escolha no controle da doença isquêmica coronariana.

Com o entendimento de todos estes mecanismos de ação que são comuns aos bloqueadores de canais de cálcio, nos permitimos afirmar que estas substâncias, além de provocar vasodilatação, protegem a perfusão coronariana e renal da paciente.

O objetivo deste trabalho é estudar a ação de um representante de um dos grupos dos bloqueadores dos canais de cálcio: o verapamil.

O verapamil, do grupo das fenilalquilaminas, claramente tem mecanismo de ação diferente das diidropiridinas. Com meia-vida de 6 horas, apresenta nível terapêutico meia hora após a ingestão do comprimido e a dose terapêutica entre 240 e $320 \mathrm{mg}$, dividida em três tomadas por dia. As diferenças entre os dois grupos farmacológicos de bloqueadores de canais de cálcio são notadas quando do uso prolongado e, especialmente, em pacientes hipertensas crônicas. As diidropiridinas mostram efeito hipotensor mais potente e rápido, o que entre gestantes não é o ideal. Além disso, os efeitos colaterais são mais comuns. Em contrapartida, as fenilalquilaminas possuem efeito hipotensor mais suave com efeitos adversos bem 
mais tolerados. O verapamil tem efeito inotrópico negativo e propriedades cronotrópicas, contribuindo para um melhor perfil hemodinâmico materno. O verapamil tem também uma ação anti-simpática e, provavelmente, efeitos no sistema nervoso central que podem aumentar a atuação antihipertensiva ${ }^{10}$.

A principal contra-indicação do verapamil está na vigência de alterações de condução cardíaca, principalmente no nodo atrioventricular ${ }^{9}$.

Em relação à teratogenicidade, Magee et al. ${ }^{11}$, em estudo realizado em 7 centros localizados no Canadá, Estados Unidos e Inglaterra com a inclusão de serviços de pediatria para o seguimento pós-natal, afirmaram, que os bloqueadores de canais de cálcio (inclusive o verapamil) não têm risco teratogênico, mesmo quando utilizados desde o primeiro trimestre da gestação. O baixo peso neonatal encontrado, por meio de regressão multivariada, foi relacionado com a doença de base que havia levado ao uso da medicação.

Estas informações sobre bloqueadores dos canais de cálcio e principalmente sobre o verapamil nos permitem admitir que utilizaremos um fármaco com mecanismo de ação claro e bem explicado. Além disto, vem se mostrando seguro para feto e recém-nato, com queda da pressão arterial materna suave e prolongada, além de poucos efeitos colaterais para a mãe. A proteção dos aparelhos cardiovascular e renal da gestante hipertensa promovida pelo verapamil é bastante atraente para o futuro clínico, com aumento da expectativa de vida da mulher.

O que nos faltava determinar era sua atuação no fluxo uteroplacentário e fetoplacentário, e por meio da análise dopplervelocimétrica deste fluxo em um grupo de gestantes hipertensas crônicas, construímos o ensaio clínico que será descrito.

\section{Pacientes e Métodos}

Este trabalho consiste de estudo longitudinal do tipo coorte, contemporâneo, randomizado, duplo-cego e placebo controlado. As pacientes envolvidas no estudo foram provenientes do Ambulatório de Pré-natal da Maternidade do Hospital Universitário Gaffrée e Guinle da Universidade do Rio de Janeiro. O protocolo do projeto foi aprovado pela Comissão de Ética em Pesquisa deste hospital, seguindo resolução número 02/84 do Conselho Regional de Medicina do Estado do Rio de Janeiro.

A população estudada foi incluída na investigação após anamnese e exame clínico detalha- dos. Como complementação foram realizados exames laboratoriais relativos à função renal (proteinúria, uréia, creatinina e ácido úrico), à função hepática (bilirrubina total e frações, transaminases, desidrogenase láctica, proteínas totais e frações), ao sistema cardiovascular (eletrocardiograma e fundo de olho) e à idade gestacional (ultra-sonografia).

Foram incluídas as gestantes portadoras de doença hipertensiva vascular crônica em sua forma leve para moderada. O diagnóstico da síndrome hipertensiva obedeceu as normas do National High Blood Pressure Education Program Working Group Report $^{4}$ que define a gestante hipertensa crônica como aquela que apresenta hipertensão antes da $20^{a}$ semana de gravidez, persistindo após o $42^{\circ}$ dia do puerpério. Ficam incluídas as pacientes que apresentarem o diagnóstico de elevação da pressão arterial prévio à gestação. Como forma leve para moderada, foram consideradas as gestantes que apresentaram niveis tensionais diastólicos $\leq 109 \mathrm{mmHg}$, além de não apresentarem alterações graves de órgãos nobres: coração, pulmão, cérebro e rins. Na vigência de niveis tensionais maiores ou comprometimento dos órgãos acima, os casos foram classificadas como graves e excluídos do estudo.

A determinação da pressão arterial foi adequada, segundo referencial teórico publicado pelo autor $^{12}$ : equipamento aneróide permanentemente aferido; braço apoiado em superficie plana ao nível do precórdio; constatado equilíbrio emocional (recomendado que seja o último procedimento da consulta); o membro utilizado será sempre o mesmo; manguito adequado ao tamanho do braço e colocado a 2 centímetros acima da prega do cotovelo; estetoscópio colocado sobre a fossa antecubital sem qualquer tipo de pressão exagerada; a insuflação deve ultrapassar 20-30 mmHg do desaparecimento do batimento audível no estetoscópio; velocidade de desinsuflação cerca de 2 a $3 \mathrm{~mm}$ $\mathrm{Hg} / \mathrm{seg} 1^{\circ}$ som de Korotkoff para a pressão sistólica; $4^{\circ}$ som de Korotkoff para a pressão diastólica.

Na vigência de hipertensão considerada grave (diastólica acima de $110 \mathrm{mmHg}$ ), a pressão arterial foi aferida após quatro horas de repouso na enfermaria.

A paciente não deveria estar em uso de nenhuma droga que tivesse efeito sobre a pressão arterial nos últimos 7 dias. A idade gestacional foi calculada pela data da última menstruação e confirmada pela ultra-sonografia, devendo estar no máximo na $24^{a}$ semana de gestação. A paciente não poderia ser portadora de qualquer patologia cardiaca que contra-indique o uso do bloqueador dos canais de cálcio (distúrbio de condução e/ou 
miocardiopatia).

Uma dopplervelocimetria foi realizada entre 26 e 28 semanas com a finalidade de avaliar o bem-estar fetal. Na vigência de centralização fetal a paciente não seria incluída no estudo. A paciente deveria manifestar por escrito, com assinatura em consentimento informado, sua concordância em participar do estudo, sendo informada corretamente a finalidade do estudo. Em qualquer momento do estudo as pacientes puderam ser afastadas por vontade própria, pelo não-comparecimento à consulta ou exame agendado, pelo uso indevido da medicação em estudo ou pelo agravamento do quadro clínico. Nas três primeiras situações as pacientes foram remarcadas para continuidade do pré-natal dentro da rotina do serviço, ao passo que na última possibilidade ficou implantado o protocolo específico da Disciplina de Obstetrícia da Universidade do Rio de Janeiro para gestantes hipertensas.

O estudo foi feito por dois observadores que permaneceram até o fim da coleta de dados: o primeiro observador foi responsável pela administração da medicação e acompanhamento da gestante; o segundo observador realizou os exames de dopplervelocimetria. O primeiro observador recebeu caixas numeradas, que continham 90 comprimidos, quantidade necessária para 30 dias de tratamento, acondicionados em blisters igualmente numerados, contendo verapamil ou placebo, randomicamente preparados em grupos de quatro: [1, 2, 3 e 4]; [5, 6, 7, e 8].

Em cada grupo dois envelopes acondicionaram comprimidos com verapamil e dois com placebo. Os comprimidos foram confeccionados com forma, volume e cor idênticos. Somente o laboratório farmacêutico tinha conhecimento da chave de randomização, responsabilizando-se pelo fornecimento da droga ativa e do placebo.

$\mathrm{O}$ primeiro observador recebeu um envelope lacrado referente ao conteúdo de cada caixa, para que pudesse abri-lo na vigência de qualquer efeito colateral e/ou complicação que acontecesse durante o estudo.

A dose utilizada de verapamil obedeceu ao Protocolo do Setor de Hipertensão da Disciplina de Obstetrícia da Universidade do Rio de Janeiro, segundo publicações já apresentadas ${ }^{13,14}$. A posologia de $240 \mathrm{mg} /$ dia, dividida em 3 tomadas diárias, foi adotada por ser a dose terapêutica em gestantes.

O exame dopplerfluxométrico foi realizado com a paciente em posição semi-sentada. Foi utilizado aparelho Toshiba modelo Echos, com transdutor de $3,5 \mathrm{MHz}$ acoplado à unidade de Doppler colorido. Com o transdutor colocado no plano longitudinal, a artéria ilíaca é visualizada na parede lateral da pelve. O transdutor foi angulado medialmente até a parede lateral do útero, até conseguir uma onda de fluxo caracterizada pela baixa pulsatilidade e alta velocidade no fim da diastóle. Para a insonação da artéria umbilical, foi calculada a média das três medidas realizadas no máximo a $3 \mathrm{~cm}$ da inserção do cordão na placenta.

O observador que realizou o exame não teve acesso ao código de randomização. Em formulário padrão anotou a relação $\mathrm{A} / \mathrm{B}$, o índice pulsátil e o indice de resistência das artérias uterinas e da artéria umbilical. Este documento recebeu o número da caixa que acondicionou a droga, para posterior análise dos resultados.

Após a primeira dopplervelocimetria, realizada entre 26-28 semanas de gestação, com finalidade de afastar fetos seriamente comprometidos (centralização), a paciente recebeu uma caixa numerada contendo 90 comprimidos, com a prescrição de utilizar 3 vezes ao dia durante 30 dias. A paciente foi agendada com 30 dias de intervalo (3032 semanas de gestação), quando foi realizada nova dopplerfluxometria de acordo com as normas já descritas. Os valores dos parâmetros analisados na dopplervelocimetria foram registrados.

A paciente deveria trazer o envelope vazio, sendo submetida a interrogatório rigoroso buscando a certeza de que a medicação foi usada corretamente. Além disso deveria relatar qualquer efeito adverso que por acaso tivesse observado. Caso o investigador colocasse em dúvida a adesão da paciente, ela seria excluída do estudo. Com o término da coleta de dados, o pesquisador teve conhecimento da chave da randomização, e pode separar os dois grupos: droga ativa e placebo.

A média e o desvio padrão dos valores dos parâmetros dopplerfluxométricos (relação A/B, índice de pulsatilidade e índice de resistência de ambas as artérias uterinas, analisadas em conjunto, e da artéria umbilical) foram calculados para os dois grupos. Para ajuizar a diferença das médias, foi utilizada a estatística $\mathrm{F}$, baseada na análise de variância, considerando significativa essa diferença quando $\mathrm{p}<0.05$.

\section{Resultados}

Entre 15 de maio de 1995 e $1^{\circ}$ de março de 1998 , entraram no protocolo de pesquisa 163 gestantes hipertensas crônicas classificadas como leve para moderada e que preencheram os critérios de inclusão, no máximo com 26 semanas de gestação.

Quarenta pacientes foram excluídas por não 
seguirem o protocolo adequadamente. As principais causas de exclusão foram: falta à consulta e/ ou exame de dopplervelocimetria (70\%), utilização equivocada da medicação, inclusive com cessão à familiar $(20 \%)$, piora do quadro da hipertensão arterial e/ou efeito colateral $(7,5 \%)$ e parto prematuro $(2,5 \%)$.

Com este número de exclusões restaram 123 gestantes que chegaram ao final do ensaio clinico, com a realização do exame de dopplervelocimetria. Os resultados desse exame foram anotados de acordo com o número da caixa de medicamento que havia sido utilizada pela paciente.

Um dos orientadores deste trabalho abriu o código de randomização, assinalando em cada protocolo de pesquisa qual a medicação que havia sido utilizada. Desta forma foram criados dois grupos: Grupo Verapamil com 61 pacientes e Grupo Placebo com 62 pacientes.

As médias, com seus respectivos desviospadrão, foram calculadas para os valores de índice de resistência, indice de pulsatilidade e relação sístole/diastóle (relação A/B), tanto para as artérias uterinas como para a artéria umbilical.

Em relação às artérias uterinas, a média e o desvio padrão foram calculados a partir da média dos valores das duas artérias de cada caso. A Tabela 1 apresenta as médias dos valores da dopplervelocimetria realizada 30 dias após a administração do placebo de forma oral e consecutiva (três comprimidos /dia). Em 62 pacientes analisadas os valores mostraram média e desvio-padrão dentro dos limites da normalidade. O mesmo procedimento foi realizado para os valores dopplervelocimétricos no grupo de gestantes submetidas ao regime terapêutico que utilizou o verapamil $(240 \mathrm{mg} /$ dia em três tomadas) na forma oral e contínua, conforme demonstrado na Tabela 2.

Calculadas as médias de cada parâmetro velocimétrico nos dois grupos estudados, foi analisada a diferença entre eles utilizando a razão $\mathrm{F}$. A Tabela 3 explicita a análise referente aos valores nas artérias uterinas. Os resultados permitem observar que não houve diferença significativa entre os registros, demonstrando a não-interferência da droga ativa na circulação uterina, avaliada pela dopplervelocimetria, no grupo estudado de hipertensas crônicas. A Tabela 4 representa a mesma diretriz analítica, mas agora avaliando a circulação fetoplacentária, lançando mão dos registros na circulação umbilical das gestantes submetidas aos dois regimes terapêuticos. Da mesma forma que na circulação uterina os resultados mostram que a utilização do verapamil não interferiu nos parâmetros dopplervelocimétricos da artéria umbilical.
Tabela 1 - Médias, com seus respectivos desvios-padrão, dos valores dos índices de resistência, pulsatilidade e da relação $A / B$ obtidos em pacientes hipertensas crônicas após a administração de 30 dias de placebo sob a forma oral e contínua.

\begin{tabular}{lccc}
\multicolumn{3}{c}{ Grupo Placebo $(\mathbf{n}=\mathbf{6 2})$} & \\
\hline & $\begin{array}{c}\text { Índice de } \\
\text { resistência }\end{array}$ & $\begin{array}{c}\text { Índice de } \\
\text { pulsatilidade }\end{array}$ & Relação A/B \\
\hline Artérias Uterinas & $0,75(0,35)$ & $1,00(0,18)$ & $2,30(0,38)$ \\
Artéria Umbilical & $0,70(0,02)$ & $1,03(0,07)$ & $3,02(0,78)$ \\
\hline
\end{tabular}

Tabela 2 - Médias, com seus respectivos desvios-padrão, dos valores dos índices de resistência, pulsatilidade e da relação $A / B$ obtidos em pacientes hipertensas crônicas após a administração de 30 dias de verapamil sob a forma oral e contínua.

\begin{tabular}{lccc}
\multicolumn{4}{c}{ Grupo Verapamil $(\mathbf{n}=\mathbf{6 1})$} \\
\hline & $\begin{array}{c}\text { Índice de } \\
\text { resistência }\end{array}$ & $\begin{array}{c}\text { Índice de } \\
\text { pulsatilidade }\end{array}$ & Relação A/B \\
\hline Artérias Uterinas & $0,82(0,28)$ & $1,06(0,12)$ & $2,42(0,51)$ \\
Artéria Umbilical & $0,73(0,12)$ & $1,04(0,13)$ & $2,94(0,32)$ \\
\hline & & &
\end{tabular}

Tabela 3 - Estudo da diferença de média entre os valores encontrados nos registros dopplervelocimétricos das artérias uterinas nos dois grupos de pacientes hipertensas crônicas, após a administração por 30 dias de placebo e verapamil sob a forma oral $e$ contínua.

Artérias Uterinas

\begin{tabular}{lccc}
\hline & $\begin{array}{c}\text { Índice de } \\
\text { resistência }\end{array}$ & $\begin{array}{c}\text { Índice de } \\
\text { pulsatilidade }\end{array}$ & Relação A/B \\
\hline $\begin{array}{l}\text { Grupo Verapamil } \\
\mathrm{n}=61\end{array}$ & $0,82(0,28)$ & $1,06(0,12)$ & $2,42(0,51)$ \\
$\begin{array}{l}\text { Grupo Placebo } \\
\mathrm{n}=62\end{array}$ & $0,75(0,35)$ & $1,00(0,18)$ & $2,30(0,38)$ \\
Valor de $\mathrm{p}$ & 0,49 & 0,39 & 0,32 \\
& $\mathrm{NS}$ & $\mathrm{NS}$ & $\mathrm{NS}$
\end{tabular}

Razão $\mathrm{F}$ aceitando significância quando $p<0.05$

Tabela 4 - Estudo da diferença de médias entre os valores encontrados nos registros dopplervelocimétricos da artéria umbilical, nos dois grupos de pacientes hipertensas crônicas após a administração por 30 dias de placebo e verapamil sob a forma oral e contínua.

Artéria Umbilical

\begin{tabular}{lccc}
\hline & $\begin{array}{c}\text { Índice de } \\
\text { resistência }\end{array}$ & $\begin{array}{c}\text { Índice de } \\
\text { pulsatilidade }\end{array}$ & Relação A/B \\
\hline $\begin{array}{l}\text { Grupo Verapamil } \\
\mathrm{n}=61\end{array}$ & $0,73(0,12)$ & $1,04(0,13)$ & $2,94(0,32)$ \\
$\begin{array}{l}\text { Grupo Placebo } \\
\mathrm{n}=62\end{array}$ & $0,70(0,14)$ & $1,03(0,07)$ & $3,02(0,78)$ \\
valor de $\mathrm{p}$ & 0,64 & 0,86 & 0,55 \\
& $\mathrm{NS}$ & $\mathrm{NS}$ & $\mathrm{NS}$ \\
\hline
\end{tabular}

Razão F aceitando significância quando $p<0.05$ 


\section{Discussão}

O primeiro ponto a ser abordado é a indicação de tratamento medicamentoso para a gestante hipertensa crônica em sua forma leve para moderada (Sociedade Internacional para o Estudo da Hipertensão na Gravidez) ou também rotulada como não-complicada ${ }^{7}$.

A conduta não intervencionista é defendida por Knuist et al. ${ }^{15}$. Em 2.413 pacientes os autores não encontraram nenhum aumento significativo de complicações maternas nos casos considerados leves que, segundo a rotina do Centro de Saúde envolvido no estudo, não recebiam nenhum tipo de medicação anti-hipertensiva.

Sibai ${ }^{16}$ conduziu ensaio clínico randomizado comparando a conduta expectante com uso de labetalol e com alfa-metildopa. Todas as pacientes tinham diagnóstico de doença hipertensiva vascular crônica leve para moderada, e entravam no protocolo no final do primeiro trimestre. Os resultados não mostraram proteção para a sobreposição de pré-eclampsia ou para o descolamento prematuro de placenta no grupo submetido aos hipotensores. As pacientes necessitavam somente de um bom pré-natal.

Uma reformulação do National High Blood Pressure Education Program foi apresentada por Mochizuki et al. ${ }^{17}$, que pode ser sintetizada da seguinte forma: “. . . poucos dados disponiveis permitam reforçar a utilização do tratamento farmacológico em pacientes hipertensas crônicas em geral, necessitando-se de mais ensaios clinicos que, infelizmente, aparecem lentamente".

A observação clínica de que este grupo de pacientes, que além da hipertensão propriamente dita tem fatores de risco acessórios, deve ser conduzido com anti-hipertensivos fica patente não só no consenso americano como também na proposta de protocolo canadense e australiana. Faltavam evidências com metodologia e estratégia adequadas $^{3,4,18}$.

Estas evidências começaram a aparecer e, durante o $11^{\circ}$ Congresso Mundial de Hipertensão na Gravidez (1998) ${ }^{17}$, Ornstein et al. ${ }^{19}$ apresentaram metanálise retirada da base de dados MEDLINE entre 1966 e 1997. Os autores compararam 14 ensaios clínicos que utilizaram a conduta não-medicamentosa (repouso, dieta, visitas domiciliares), com 23 que lançaram mão de uma droga anti-hipertensiva. Por meio da análise de risco observaram semelhante proteção para a hipertensão (odds ratio $=0.38 /$ odds ratio $=0.31$ ), sem impacto nas taxas de crescimento intrauterino retardado e de proteinúria. O principal beneficio registrado nos trabalhos que favoreci- am o uso do anti-hipertensivo foi o percentual de pacientes internadas por agravamento (odds ratio $=0,77 /$ odds ratio $=0.31)$ : as pacientes que usavam algum fármaco necessitavam menos da hospitalização prévia ao parto que o grupo sem intervenção.

$\mathrm{O}$ aspecto seguinte é a repercussão perinatal da hipertensão. O conceito antigo que admitia que a gestante hipertensa crônica, sem a sobreposição de pré-eclampsia, apresentava os mesmos resultados perinatais que a gestante normotensa não encontra eco na literatura nacional e internacional da atualidade. MacCowan et al. ${ }^{20}$, analisando 155 gestantes hipertensas crônicas neozelandesas, registraram incidência de 10,9\% de recémnatos pequenos para a idade gestacional neste grupo, ao passo que no grupo controle essa incidência foi de $4,1 \%$. Análise de risco com odds ratio $=2.9(1.6-5.0)$ foi calculada. A prematuridade e a perda perinatal não se mostraram significativamente diferentes nos dois grupos.

Com essa ótica como hipótese de pesquisa apareceram alguns importantes trabalhos mostrando, como primeira preocupação, o controle da hipertensão arterial e conseqüente proteção materna. O crescimento intra-uterino retardado e a mortalidade perinatal são, invariavelmente, usados como marcadores do feto e recém-nato. Mais recentemente, em cinco ensaios controlados, a alfa-metildopa foi comparada com placebo ou com não medicação ${ }^{16}$. Três deles mostraram resultados semelhantes entre os dois grupos, tanto no enfoque materno como no perinatal. Dois ensaios demonstraram aumento significativo da idade gestacional no momento do parto no grupo droga. Somente um trabalho mostrou um aumento no peso fetal no grupo estudo. O mais importante é que nenhum dos cinco trabalhos mostrou diferenças quanto ao tópico sobreposição de pré-eclampsia. Os resultados com a alfa-metildopa repetiram-se em nosso trabalho. Como era de se esperar, a incidência de pré-eclampsia foi idêntica (16 casos em cada grupo), ratificando a idéia hoje aceita universalmente: o tratamento clínico de gestantes hipertensas crônicas não é profilático para o aparecimento da pré-eclampsia.

Já de muito tempo vem se estudando o uso do atenolol no controle das síndromes hipertensivas. Dumez et al. ${ }^{21}$ referem casos de bloqueio cardíaco com utilização do atenolol. Este talvez tenha sido o primeiro sinal de alerta para a não-preferência por este $\beta$-bloqueador, conduta esta que vem se fortalecendo. Montan et al. ${ }^{22}$, em trabalho bem elaborado, observaram que o atenolol aumentava a resistência vascular uteroplacentária e fetoplacentária. O estudo comparava os mesmos parâmetros em pacientes submetidas ao pindolol, 
concluindo que este efeito deletério não acontecia com o segunda droga.

Mais recentemente Lip et al. ${ }^{23}$ publicaram estudo prospectivo de 16 anos (1980-1995) na cidade de Birmingham (Inglaterra). Entre as 398 gestantes hipertensas atendidas consecutivamente neste periodo, 76 utilizaram atenolol e 109 fizeram uso de outras drogas anti-hipertensivas. Os recém-natos submetidos ao atenolol apresentaram peso menor, peso placentário mais baixo e indice ponderal menor. Apesar de tratar-se de estudo passivel de crítica, pois não foi adequadamente desenhado, a análise de resultados, inclusive com regressão multivariada, permite uma conclusão concordante com a literatura: o atenolol interfere no fluxo uteroplacentário com conseqüente baixo peso ao nascer.

Mesmo com a literatura aceitando o pindolol e a alfa-metildopa como drogas seguras, a revisão bibliográfica sobre o uso de anti-hipertensivos em gestantes hipertensas crônicas na década de 90 começou a mostrar uma preferência pelos bloqueadores dos canais de cálcio. A baixa efetividade da hidralazina oral sobre a pressão arterial materna, as dificuldades causadas por alguns $\beta$-bloqueadores no resultado perinatal somadas aos conhecimentos farmacológicos e clínicos dos bloqueadores dos canais de cálcio levaram a esta mudança de rumo no tratamento clínico da gestante hipertensa crônica.

Vários estudos começaram a aparecer com os mais variados tipos de bloqueadores dos canais de cálcio. Alguns exemplos podem ser citados a título de informação. Brogden e Sorkin ${ }^{24}$ publicaram consistente trabalho com a utilização da isradipina entre 29 e 36 semanas de gestação, quer sob a forma oral contínua como também sob a forma venosa utilizada na emergência hipertensiva. A administração oral de 5 a $10 \mathrm{mg}$ da isradipina por dia durante várias semanas mostrou um controle na pressão arterial sem alterações na velocidade de fluxo nas artérias uterinas e na artéria umbilical, medidas pelo índice de pulsatilidade.

Uma outra contribuição sobre comparação de efeitos sobre o feto com a utilização de um bloqueador de canais de cálcio foi dada por Alves ${ }^{25}$, que em tese de doutorado comparou a isradipina com o atenolol. Os resultados desse importante trabalho nacional na área mostraram melhora no fluxo placentário medido pela dopplervelocimetria de vasos maternos e fetais no grupo submetido à isradipina. O controle da pressão arterial foi semelhante para as duas drogas. A comparação com nosso estudo fica prejudicada pois todos os tipos de hipertensão foram arrolados no trabalho, apesar de que $85 \%$ dos casos eram de gestantes hipertensas crônicas. No entanto a idéia é a mesma: melhorar o estado materno sem prejuízo no território fetal.

Mais recentemente, a literatura internacional publicou outra contribuição sobre a utilização dos bloqueadores dos canais de cálcio em gestantes que cursaram com pressão diastólica abaixo de $110 \mathrm{mmHg}$. O Gruppo di Studio Ipertensione in Gravidanza ${ }^{26}$ lançou mão da nifedipina para seu estudo. Com ensaio clínico randomizado, administraram a droga para 145 gestantes e placebo para 138 pacientes. Os resultados perinatais e maternos (prematuridade, taxa de cesarianas, peso do recém-nato e admissão na unidade terciária) foram melhores no grupo tratado. Este estudo, em nossa opinião, incorreu em erro metodológico ao reunir gestantes com hipertensão crônica e com pré-eclampsia leve em um só contingente, mas mesmo assim ratificou bons resultados com o bloqueador dos canais de cálcio.

O objetivo deste trabalho foi utilizar uma destas drogas vasodilatadoras, o verapamil, que além de demonstrar controle efetivo da pressão arterial materna demonstrou não exercer nenhuma ação na circulação uteroplacentária e fetoplacentária. Estudos com o verapamil já foram tentados durante o ciclo gravídico-puerperal. Marlettini et al. ${ }^{27}$ cotejaram o verapamil com dois $\beta$ - bloqueadores: o pindolol e o atenolol. Os resultados obtidos demonstraram uma similar atuação em relação ao pindolol e melhores índices nos parâmetros de crescimento fetal e prematuridade com o verapamil, na comparação com o atenolol.

O verapamil, utilizado por Belfort et al. ${ }^{28}$, já havia se mostrado efetivo no controle da pressão arterial sem afetar o fluxo uteroplacentário e fetoplacentário. A utilização do fármaco, no entanto, foi feita sob a forma endovenosa para controle da pré-eclampsia grave. A dopplervelocimetria mostrou, inclusive, o desaparecimento da incisura nas artérias uterinas. Este estudo, ao analisar a circulação materna e fetal em gestantes hipertensas crônicas, admitiu que este efeito pudesse acontecer em qualquer tipo de síndrome hipertensiva da gestação. Em nosso estudo a utilização do verapamil não apresentou modificações na circulação uteroplacentária e fetoplacentária. Com valores iguais para indices de resistência e pulsatilidade e relação sístole/diástole nos dois grupos estudados, concluímos que o fármaco não exerceu nenhuma repercussão sobre o fluxo placentário. A preservação do fluxo placentário demonstrada com os resultados de nosso ensaio clínico, é ratificada quando complementamos as informações com dados provenientes do acompanhamento das pacientes protocoladas até o final 
da gestação.

O presente trabalho ratificou algumas premissas que devem nortear o profissional de saúde de qualquer especialidade ou segmento. O diagnóstico definitivo de uma doença é somente o primeiro estágio para o atendimento de um paciente. Logo em seguida ele deve escolher o mais seguro e efetivo tratamento a ser administrado. Enquanto estudos observacionais são adequados para análise de risco de doenças, um estudo clínico controlado e randomizado é imperativo para comparar e conseqüentemente escolher a melhor opção terapêutica ${ }^{29}$.

O ensaio clínico em tela transformou a sensação de um grupo de saúde em sua experiência prática de mais de quinze anos em observação metodologicamente aceita como verdadeira. O acaso não mais pode ser responsabilizado pelos resultados atingidos. A gestante hipertensa crônica pode ter seu quadro clínico e laboratorial controlado durante a gestação com o emprego do verapamil. Esta afirmativa já se faria evidente por clínicos e cardiologistas, mas uma possivel repercussão negativa sobre o território placentário, influenciando o fluxo sangüíneo uterino e fetal, não podia ser comprovada ou rejeitada, à luz de evidências científicas.

Este trabalho demonstra que não existe diminuição no fluxo sangüíneo com o uso crônico do verapamil. O que nos falta comprovar é a segurança do verapamil no seguimento a longo prazo da criança submetida ao regime terapêutico intraútero. Em relação aos bloqueadores de canais de cálcio, em geral, o curto tempo de utilização no ciclo gravídico-puerperal ainda não permitiu acompanhamento adequado destas crianças. Somente a alfa-metildopa foi corretamente estudada em elegante trabalho de Redman ${ }^{30}$. Nesta pesquisa com crianças até a idade escolar, não foi demonstrada intercorrência clínica que pudesse ser relacionada com o uso do fármaco durante o período gestacional e lactação.

Impossivel deixar de concluir sobre o benefício na utilização do verapamil em gestantes hipertensas crônicas na forma leve para moderada, pois não só controlaremos a gestação em curso como também exerceremos uma proteção efetiva no futuro clínico desta mulher. Isto tudo sem nenhuma repercussão no pequeno paciente que, ainda intra-útero, é de total responsabilidade do obstetra.

Neste aspecto, por que não admitir que esta conduta deva ser rotineira em países em desenvolvimento que, enfrentando problemas de saúde pública muito sérios e de difícil solução a curto prazo, não podem fornecer um atendimento abrangente e universal?

\section{SUMMARY}

Purpose: this study, using verapamil, a slow calcium channel blocker, was a randomized, clinical, double blind and placebo controlled trial, whose objective was to observe if there was a uteroplacental and fetoplacental flow variation during its chronic oral use.

Methods: 123 patients were accompanied: study group $(n=$ 61), submitted to verapamil $240 \mathrm{mg} /$ day and control group $(n=62)$, submitted to placebo. These patients were randomized into groups of four women and treatment or placebo was given for thirty days. A flow examination of the uterine arteries and umbilical artery through dopplervelocimetry was recorded. The values of resistance (RI) and pulsatility index (PI) and of the systole/diastole ratio $(S / D)$ of the arteries were compared after the drug administration calculating means and standard deviations.

Results: the verapamil group showed $R I=0.82(0.28), P I=$ $1.06(0.12)$ and $S / D=2.42(0.51)$ in the uterine arteries. The placebo group showed $R I=0.75$ (0.35), $P I=1.00$ (0.18) and $S / D=2.30(0.38)$. When we analyzed the umbilical artery, the verapamil group showed $R I=0.73$ (0.12), $P I=$ 1.04 (0.13) and $S / D=2.94$ (0.32). The placebo group showed $R I=0.70(0.14), P I=1.03(0.07)$ and $S / D=3.02(0.78)$. The statistical analysis of the differences of the means by the $F$ ratio showed that there was no difference between these two groups.

Conclusion: this study indicates the use of verapamil for chronic hypertensive pregnants since it does not provoke damage to the uterine and fetal blood flow.

KEY WORDS: Hypertension in pregnancy. Verapamil. Placental flow. Dopplervelocimetry

\section{Referências}

1. Henriksen T. Hypertension in pregnancy: use of antihypertensive drugs. Acta Obstet Gynecol Scand 1997; 76:96-106.

2. Kaplan NM. Clinical hypertension, 6th edn. Baltimore: Williams \& Wilkins; 1994.

3. Moutquin JM, Garner PR, Burrows RF, et al. Report of the Canadian Hypertension Society Consensus Conference. 2. Nonpharmacologic management and prevention of hypertensive disorders in pregnancy. CMAJ 1997; 157:907-19.

4. National Blood Pressure Education Program Working Group Report on High blood pressure in pregnancy. Am J Obstet Gynecol 1990; 163:1691712 . 
5. Management of hypertension in pregnancy: executive summary. Australasian Society for the Study of Hypertension in Pregnancy. Med J Aust 1993; 158:700-2.

6. Jain L. Effect of pregnancy and chronic hypertension on pregnancy outcome. J Perinatol 1997; 17:425-7.

7. Kahhale S, Zugaib M. Sindromes hipertensivas na gravidez. $1^{\mathrm{a}}$ edn. Rio de Janeiro: Atheneu; 1995.

8. Lindheimer MD, Roberts JM, Cunningham FG. Hypertensive disorders in pregnancy. $2^{\text {nd }}$ edn. Connecticut: Library of Congress; 1999.

9. Fisher M, Grotta J. New uses for calcium channel blockers. Therapeutic implications. Drugs 1993; 46:961-75.

10.Frishman WH, Michaelson MD. Use of calcium antagonists in patients with ischemic heart disease and systemic hypertension. Am J Cardiol 1997; 79:33-8.

11. Magee LA, Schick B, Donnenfeld AE, et al. The safety of calcium channel blockers in human pregnancy; a prospective multicentre cohort study. Hypertens Pregnancy 1997; 16:138-41.

12.Vasconcellos MJA, Ritto MNG, Vianna MA, Louro EP, Rocco R. Como aferir a pressão arterial durante a gestação. Rev Ginecol Obstet 1991; 2:176-9.

13.Vasconcellos MJA, Ritto MNG, Lopes LM, Louro EP, Rocco R. Utilização dos bloqueadores dos canais de cálcio no controle das síndromes hipertensivas da gravidez. Rev Ginecol Obstet 1993; 4:63-9.

14.Vasconcellos MJA, Ritto MNG, Oliveira ILC, Louro EP, Rocco R. Resultados do protocolo de acompanhamento da gestante hipertensa. Rev Ginecol Obstet 1994; 5:22-8.

15.Knuist M, Bonsel GJ, Zondervan HA, Treffers PE. Intensification of fetal and maternal surveilance in pregnant women with hypertensive disorders. Int J Gynaecol Obstet 1998; 61:127-33.

16.Sibai B M. Chronic hypertension in pregnancy. Clin Perinatol 1991; 18:833-44.

17.Mochizuki M, Satoh K, Ogita S. Kobe Report. Report of the 11 th World Congress of the International Society for the Study of Hypertension in Prenancy (ISSHP); 1998; Kobe, Japan. 1st. ed Kobe: Society for the Study of Toxemia of Pregnancy; 1998. p.110.

18. Rey E, LeLorier J, Burgess E, Lange IR, Leduc L. Report of the Canadian Hypertension Society Consensus Conference. 3. Pharmacologic treatment of hypertensive disorders in pregnancy. CMAJ 1997; 157:1245-54.
19. Ornstein M, von Dadelszen P, Magee L A. Risks \& benefits of pregnancy hypertension management - overview of the RCTs. Annals of the 11 th World Congress of International Society for the Study of Hypertension in Prenancy (ISSHP); 1998; Kobe, Japan. 1st ed. Kobe: Society for the Study of Toxemia of Pregnancy; 1998. p.105.

20.MacCowan LM, Buist RG, North RA, Camble G. Perinatal morbidity in chronic hypertension. $\mathrm{Br}$ J Obstet Gynaecol 1996; 103:123-9.

21.Dumez Y, Tchobroutsky C, Hornych H, Amiel-Tison C. Neonatal effects of maternal administration of acebutolol. Br Med J 1981; 283:1077-9.

22. Montan S, Liedholm H, Lingman G, Marsal, K, Sjoberg NO, Solum T. Fetal and uteroplacental haemodynamics during short term atenolol treatment in pregnancy hypertension. Br J Obstet Gynaecol 1987; 94:312-7.

23.Lip GYH, Beevers M, Churchill D, Shaffer LM, Beevers DG. Effect of atenolol on birth weight. Am J Cardiol 1997; 79:1436-8.

24.Brogden RN, Sorkin EM. Isradipine. An update of its pharmacodynamic and pharmacokinetic properties and therapeutic efficacy in the treatment of mild to moderate hypertension. Drugs 1995; 49:618-49.

25.Alves EA. Estudo prospectivo, comparativo da isradipina e atenolol no tratamento de gestantes hipertensas [tese]. São Paulo: Universidade de São Paulo; 1998.

26.Nifedipine versus expectant management in mild to moderate hypertension in pregnancy. Gruppo di Studio Ipertensione in Gravidanza. $\mathrm{Br} \mathrm{J}$ Obstet Gynaecol 1998; 105:718-22.

27.Marlettini MG, Crippa S, Morseli-Labate AM, Contarini A, Orlandi C. Randomized comparision of calcium antagonists and beta-blockers in the treatment of pregnancy induced hypertension. Curr Ther Res 1990; 48:684-94.

28.Belfort M, Akovic K, Anthony J, Saade G, Kirshon $\mathrm{B}$, Moise $\mathrm{K} \mathrm{Jr}$. The effect of acute volume expansion and vasodilatation with verapamil on uterine and umbilical artery Doppler indices in severe preeclampsia. J Clin Ultrasound 1994; 22:317-25.

29.Knapp RG, Miller III MC. Clinical epidemiology and eiostatistics. $1^{\text {st }}$ edn. Baltimore: Williams \& Wilkins; 1992.

30.Redman CWG. Hypertension in pregnancy In: De Swiet M, editor. Medical Disorders in Obstetric Practice. $1^{\text {st }}$. ed. London: Blackwell Science; 1995. 